\title{
The Frequency and Factor Structure of Obsessional Intrusive Thoughts in College Students Group
}

\author{
Seung-Ah Jung1)
}

\begin{abstract}
The purpose of this paper is to identify the universality of obsessional intrusive thoughts in Korean normal people, and to examine the sub-structure of the intrusive thoughts through factor analyzing ROII (Revised Obsessional Intrusive thought Inventory) items. Additionally, sex differences across ROII sub-factors were tested. The result supported the universality of intrusive experience, in that $86 \%$ of 377 normal college students reported at least one item of ROII. Through factor analysis, 6 sub-factors (aggression, accidents, sex, checking, contamination, loss of self-control) were identified, which were generally consistent with previous foreign studies on the factor structure of intrusive thinking. And subsequent $t$-test across those 6 factors showed significant sex differences in aggression (male $>$ female), sex(male $>$ female), checking(male $<$ female), contamination(male $<$ female) factors. Implications of these results and limitations of this study were discussed.
\end{abstract}

Keywords : Factor Structure, Frequency, Intrusive Thought, ROII, Sex Difference.

\section{Introduction}

Obsessive Compulsive Disorder(OCD) is a relatively common psychiatric disorder in which $0.7-2.5 \%$ of the general population is experiencing once in a lifetime[1][2], and in Korea, recent epidemiological data report $0.6 \%$ of lifetime prevalence[3]. The main symptoms of OCD are 'compulsions' defined as repetitive and ritualistic behaviors that occur in response to an 'obsession' defined by unwanted repetitive thought, images, and impulses[4]. An obsession is an intrusive, irrational ideas(impulses or images) that float on consciousness, irrespective of one's will, whose contents usually are unpleasant or threatening that the person does not want to think. For example, ideas that you or someone you are intimate with may have a terrible accident, commit some aggressive and unethical behavior that you can not control yourself, or you may least to sudden sexual behavior in a situation that is not at all appropriate. Patients with $\mathrm{OCD}$ often repeat a variety of behaviors in order to resist these thought or prevent them from being put into practice. This is a compulsion.

Received(July 21, 2018), Review Result(1st: August 1, 2018, 2nd: August 31, 2018), Accepted(September 10, 2018)

1) (Professor) 61452 Dept. Counselling Psychology, Chosun Univ., 309 Pilmun-Daero, Don-Gu, Gwangju, Korea email: jsa@chousn.ac.kr 
According to the cognitive-behavioral model explaining the pathological mechanisms for the development and maintenance of OCD, the clinical symptoms of OCD patients and the OCD-like symptoms of normal populations are only quantitatively different on a degree of symptom continuum, not qualitatively different. In other words, the difference between OCD patients and normal is determined by how they respond to the obsessions rather than to the content or intensity of the obsessions. Earlier, Rachman and De Silva[5] have reported pioneering research that the intrusive obsessional thoughts are also experienced by normal people, and these thoughts were not different with OCD's in the contents or frequency. Although there have been some critical studies these results since then, subsequent studies have largely supported this. For example, in the Rachman and De Silva study[5], the incidence rate of intrusive thought experienced by the general population was $80 \%$, and a recent study of 777 patients from 15 cities in 13 countries[6][7] was 84-100\%. However, some studies have suggested that there may be cultural differences in the prevalence of OCD or in the distribution of detailed contents of intrusive thought. Most of the evidence supporting the universality of intrusive thought came from Western studies in Western cultures, and there is still a lack of basic data or empirical studies as to whether the cognitive-behavioral model based on such evidences can be applied to oriental cultures, especially to Koreans.

The purpose of this study is to investigate whether Western studies that obsessional intrusive thoughts, which are one of the key symptoms of OCD, are common among the general population can be supported by normal people in Korea. If, as in the West, intrusive thought is universally experienced in the Korean general population, there arise other related questions. Are the contents of the intrusive thoughts similar to those reported in Western studies or are there any specific aspects other than that of the West? Is there gender difference in the incidence and contents of intrusive thoughts? This study will answer these subsequent questions.

\section{Method}

\subsection{Subject}

A questionnaire survey was conducted on 400 male and female college students. The data of 337 respondents were analyzed except for the 23 respondents whose answers were judged to be unfaithful because of too many non-responses or repeated same responses. The sex ratio of 377 subjects was male: female $=37.2 \%$ : $62.87 \%$, and the mean age was $22.03(\mathrm{SD}=1.98)$ : 


\subsection{Measurement Tools and Analysis Method}

ROII (Revised Obsessive Intrusions Inventory) developed by Purdon and Clark[8] for the purpose of measuring obsessional intrusive thoughts in non-clinical groups was used. This self-reporting survey tool was comprised of 52 items, measuring the degree of intrusive thoughts experienced by subjects on 7 points scale $(0$ : not at all - 6: several times a day). In this study, Korean version of ROII translated by Lee[9] was used. The internal consistency coefficient(a) reported by the original authors was .93. In this study alpha was .96. All statistical analysis conducted in this study were by SPSS(Ver.23).

\section{Results}

\subsection{Frequency of Experiencing Intrusive Thoughts}

Of the total 377 subjects analyzed, 53(14.5\%) were subjects who did not experience any type of intrusive thought at all, ie the total score of ROII was 0. That is to say, regardless of the content of the thought, it was found that approximately $86 \%$ of the respondents reported experiencing more than one intrusive thought. These results are within the range of the incidence rate of the intrusive thoughts reported by previous Western studies. This also suggests that experiencing intrusive thought itself in Korean non-clinical groups is universal rather than pathological or rare phenomenon.

\subsection{Factor Structure of ROII and Sex Differences Across Factors}

In order to investigate the content categories of intrusive thoughts experienced by subjects, a factor analysis of ROII items was conducted. The results of $\mathrm{KMO}$ (Kaiser-Meyer-Olkin) test(.915) and Bartlett's test of sphericity $(p=.000)$ confirmed that the sample of this study was appropriate for factor analysis. Thus factor analysis was conducted by using principal axis factoring and with the factor rotation method of direct oblimin $($ delta $=0)$ because the correlation between factors was expected.

According to the original authors, in ROII, a single factor for males and two factors for females were derived. But because this study was intended to search the factor structure without any assumptions about the number of factors, the researcher of this study first 
examine factors only on the base of Eigen value $>1$, and identify 11 factors. It then went through a three-step process to examine whether these 11 factors could be reduced to more obvious factors. Fist, reviewing the scree chart, it was found that the explanatory volume no longer increases obviously after five factors. Second, the researcher identified factor loadings of each items across 11 factors less than .40 in the pattern matrix. Third, the similarity of item content was compared between those items with factor loadings of .40 or less and above .40. Through this process, the researcher concluded that summarizing the six factors is the most appropriate factor structure to show detailed structure while minimizing the loss of information. Finally, another factor analysis was conducted in the same way by specifying the number of six factors determined through this process.

As a result, it was confirmed that the 52 items of ROII could be classified into much more clear categories of content. But only three items(15. 'I have ever thought of rudely speaking or insulting a strangers', 24. 'I have ever thought that the faucet was opened to flood the water', 40. 'I have ever thought of being a homosexual or having homosexual love'.)were still difficult to classify clearly into any of the six factors, and these three items were not included in the following analysis about the sex differences. The six factors selected by this process were aggression, contamination, checking, sex, accident(disaster, death), and loss of control. The result of this factor analysis and the internal consistency coefficient(a) of the items belonging to each factor are presented in [Table 1]. In addition, t-test(bilateral test) was conducted to identify whether there were sex differences across the six thema of intrusive thoughts thus identified. The result of this t-test showed that male scores were higher in 'sex' and 'accident', female scores were higher in 'checking' factors, though there were no significant differences in the total score. The results of this analysis are shown in [Table 2].

\section{Conclusion}

The cognitive-behavioral model explaining the occurrence, formation and maintenance of OCD was established as the current cognitive model stimulated by the pioneering research results of Rachman and De Silva[5]. According to this model, experiencing the intrusive thought itself is not pathological, and it is determined whether or not it will develop into an OCD based on how an individual interprets and tries to control such intrusive thought in subsequent cognitive processes. In Addition, the prevalence rate of OCD is stable across cultures, but some previous studies have suggested that the subtypes of OCD can be affected by culture or gender. Therefore, it is necessary to study whether the research results accumulated in the Western 
culture can be applied to Koreans, and if applicable, what they have in common and what the differences are. This study was intended to investigate whether the results of the Western study that the initial cognitive process assumed in such a cognitive model of OCD, that is, the experiences of intrusive thought are common in the general population, are also the same in Koreans. Another important purpose of this study is to confirm whether the factor structure of the intrusive thought is also substantially the same as that revealed in Western studies.

For this goal, the response of self-report questionnaire assessing intrusive thoughts were analyzed for normal college students and it was confirmed that the frequency and the factor structure of intrusive thoughts were similar to those reported in Western literature. First, even though the age group is limited to the twenties, it was found that intrusive thoughts are common phenomenon even in the normal group, so that $86 \%$ of the subjects of this study showed positive response to at least one item describing unwanted intrusive thought.

The results of this study have specific implications in that, ROII, which was used in this study is a tool to measure only OCD-specific intrusive thoughts other than other forms of intrusive thoughts like 'worry', 'apprehension' or 'rumination' which were found in other mental disorders including depression, generalized anxiety disorder, phobic patients. First, it has a therapeutic implication in the context of prevention, in that, though the obsessional intrusive thought, an idea that can hardly be presented openly to others and seems to be pathological, the thought reported by OCD is a universal phenomenon that normal people also experience. In the school or counseling scene adolescents who are stick to and have troubled with intrusive thought can easily be found. The results of this study can serve as an objective basis for assuring them that they will not fall into a more serious clinical condition due to this intrusive thought in the future and can help them flexibly deal with their inner experiences. Second, it was confirmed that the major content domains of intrusive thoughts revealed through factor analysis of ROII are roughly matched with major subtype of OCD symptoms including compulsions. The obsessions or compulsions associated with contamination and checking, harm and disaster are the most frequently observed OCD symptoms, and in addition obsessions related with sexual ideas or rituals and losing control or going crazy are another symptom subtype that can commonly be observed by clinicians. In this study the types of intrusive thoughts were also categorized as such. This suggests that cultural differences have little effect on intrusive thought, and it also shows the possibility of a close relationship between the type of intrusive thought and the OCD symptoms. Therefore, if empirical studies can show whether there is actually a close relationship between specific intrusive thought and specific OCD symptoms, a cognitive model explaining OCD will prove also appropriate for 
Koreans. Third, in the experience of intrusive thought, it was found that there were sex differences at some categories of six factors. While male subjects experienced more likely aggression and sex related intrusions than females, female subject experienced more likely contamination and checking related intrusions than males. This suggests the possibility that there is a sex difference in the frequency of the experience according to some themes of intrusive thought, and this subsequently affects the sensitivity to those intrusions or the way of coping with the intrusions. In the future, if a study can confirm the sex difference in coping strategies for specific intrusive thought, it may be necessary to develop a differential treatment program considering the results of this study in treating OCD.

Although this study obtained results that can give some above implications, it has some limitations. First of all, it can-not be sure that similar results will be seen in younger and older adults because the subjects participated in this study were limited group of people in their early 20s. In addition, although it was aimed to know the frequency and contents of intrusive thought in the normal group, results that can be compared with the clinical group actually diagnosed with OCD should be derived for more sound ground for applying cognitive model of OCD to general population. Because it is necessary to compare directly whether the intrusive thought will be experienced at a similar frequency and degree irrespective of whether they are diagnosed patients or normal people.

[Table 1] Factor Structure of ROII

\begin{tabular}{l|ccccc}
\hline & \multicolumn{4}{c}{ Factor } \\
\hline 21. Stabbing family member & 1 & 2 & 3 & 4 & 5 \\
13. Pushing family - train, car & $\mathbf{. 8 2 4}$ & .014 & .077 & .008 & -.102 \\
34. Holding up bank & $\mathbf{. 8 2 3}$ & -.018 & -.092 & .052 & .095 \\
10. Fatally pushing friend & $\mathbf{. 7 3 6}$ & .103 & .006 & -.161 & -.137 \\
20. Choking family member & $\mathbf{. 7 3 5}$ & -.064 & -.097 & .126 & .108 \\
12. Pushing stranger - train, car & $\mathbf{. 7 2 9}$ & .063 & -.051 & .042 & -.113 \\
19. Hurting family & $\mathbf{. 6 5 7}$ & -.023 & .040 & .341 & .160 \\
33. Grabbing money & $\mathbf{. 5 4 0}$ & .080 & .099 & .048 & -.186 \\
16. Bumping into people & $\mathbf{. 5 3 6}$ & .056 & -.102 & -.059 & -.232 \\
9. Fatally pushing stranger & $\mathbf{. 5 2 0}$ & .022 & -.253 & .234 & -.014 \\
27. Throwing something & $\mathbf{. 5 2 0}$ & .006 & -.041 & .402 & .031 \\
18. Insulting family & $\mathbf{. 5 1 4}$ & .084 & -.241 & -.055 & -.215 \\
14. Hurting strangers & $\mathbf{. 3 9 9}$ & .138 & -.029 & .026 & -.222 \\
17. Insulting authority & $\mathbf{. 3 7 3}$ & .045 & -.148 & .336 & -.053 \\
& $\mathbf{. 3 3 2}$ & .136 & -.184 & .152 & -.145
\end{tabular}




\begin{tabular}{|c|c|c|c|c|c|}
\hline 47. Contamination - phones & -.030 & .872 & -.007 & .055 & .104 \\
\hline 46. Contamination - doors & -.008 & .826 & .064 & -.013 & -.014 \\
\hline 49. Transmit fatal disease & .161 & .786 & -.066 & -.125 & .114 \\
\hline 50. Everything away & -.038 & .757 & -.059 & -.004 & -.053 \\
\hline 45. Catching STD & -.026 & .751 & -.092 & -.027 & .093 \\
\hline 51. All dust off floor & -.044 & .741 & .000 & -.009 & -.139 \\
\hline 48. Fatal disease - strangers & .215 & .735 & -.083 & -.046 & .118 \\
\hline 52. Dirt in unseen places & -.117 & .703 & -.042 & .076 & -.130 \\
\hline 23. Home unlocked, intruder & -.075 & $(.392)$ & .117 & .331 & -.198 \\
\hline 22. Heat/stove on, accident & -.079 & $(.346)$ & .107 & .266 & -.189 \\
\hline 41. Authority figure, naked & .082 & .071 & -.892 & -.029 & .186 \\
\hline 42. Strangers, naked & .044 & .042 & -.857 & .010 & .172 \\
\hline 43. Sex in public & -.102 & -.042 & -.846 & .026 & -.092 \\
\hline 36. Sex with authority figure & .100 & .049 & -.764 & -.037 & .004 \\
\hline 38. Kissing authority figure & .076 & .056 & -.716 & -.054 & -.043 \\
\hline 35. Sex with unwanted person & -.117 & .049 & -.637 & .028 & -.147 \\
\hline 37. Fly/blouse undone & -.170 & .126 & -.548 & .097 & -.241 \\
\hline 44. Disgusting sex act & .021 & -.036 & -.528 & .157 & -.227 \\
\hline 39. Exposing myself & .115 & .003 & -.491 & .244 & -.005 \\
\hline 25. Swearing in public & .343 & .028 & -.358 & -.035 & -.327 \\
\hline 15. Insulting strangers & .291 & .048 & -.291 & .235 & -.169 \\
\hline 1. Driving into window & .027 & .079 & .061 & .823 & .145 \\
\hline 2. Running car off road & -.157 & .007 & -.003 & .820 & -.056 \\
\hline 4. Swerving into traffic & -.063 & .021 & -.031 & .725 & -.093 \\
\hline 3. Hitting animals, people & .026 & -.037 & -.002 & .703 & .001 \\
\hline 5. Smashing into objects & .167 & -.057 & -.094 & .629 & .040 \\
\hline 7. Cutting off finger & .168 & .014 & -.106 & .534 & -.052 \\
\hline 6. Slitting wrist/throat & .096 & .055 & -.063 & .524 & -.038 \\
\hline 8. Jumping off high place & .011 & -.018 & -.229 & .510 & -.115 \\
\hline 11. Jumping - train, car & .175 & .016 & -.115 & .454 & -.066 \\
\hline 24. Taps left on, flood & .179 & .238 & .134 & .362 & -.088 \\
\hline 31. Wrecking something & .192 & .037 & -.117 & .079 & -.633 \\
\hline 30. Breaking window & .172 & .046 & -.059 & .170 & -.600 \\
\hline 29. Scratching car paint & .173 & .014 & -.168 & .116 & -.572 \\
\hline 32. Shoplifting & .402 & .025 & -.197 & -.046 & -.450 \\
\hline 26. Breaking wind in public & .184 & .067 & -.110 & .038 & -.406 \\
\hline 28. Causing a public scene & .232 & .156 & -.060 & .041 & -.404 \\
\hline 40. Acts against sex preference & -.028 & .165 & -.228 & .179 & -.264 \\
\hline
\end{tabular}

Note. Item No.23 and 24 were categorized as other factor, because of their contents and low factor loadings 
The Frequency and Factor Structure of Obsessional Intrusive Thoughts in College Students Group

[Table 2] Sex Difference According to Factor Structure of ROII

\begin{tabular}{|c|c|c|c|c|c|c|}
\hline \multicolumn{2}{|c|}{ Factors of ROII } & $\mathrm{N}$ & Mean & SD & $\mathrm{t}$ value & Cronbach's a \\
\hline \multirow[t]{2}{*}{ Aggression(other, self) } & $\mathrm{M}$ & 139 & 4.14 & 8.376 & \multirow{2}{*}{$2.411^{*}$} & \multirow{2}{*}{.925} \\
\hline & $\frac{F}{M}$ & $\frac{237}{130}$ & 2.40 & 5.650 & & \\
\hline \multirow{2}{*}{ Contamination } & $\mathrm{M}$ & 139 & 4.63 & 8.013 & \multirow{2}{*}{-.896} & \multirow{2}{*}{.923} \\
\hline & $\mathrm{F}$ & 238 & 5.37 & 7.705 & & \\
\hline \multirow{2}{*}{ Checking } & M & 138 & 2.11 & 2.794 & \multirow{2}{*}{$-1.968 *$} & \multirow{2}{*}{.813} \\
\hline & $\mathrm{F}$ & 238 & 2.69 & 2.766 & & \\
\hline \multirow{2}{*}{ Sex } & $\mathrm{M}$ & 139 & 3.47 & 6.461 & \multirow{2}{*}{$2.832 * *$} & \multirow{2}{*}{.916} \\
\hline & $\mathrm{F}$ & 238 & 1.71 & 4.627 & & \\
\hline \multirow{2}{*}{ Accident(harm, death) } & M & 138 & 5.11 & 6.936 & \multirow{2}{*}{.109} & \multirow{2}{*}{.887} \\
\hline & $\mathrm{F}$ & 238 & 5.03 & 6.748 & & \\
\hline \multirow{2}{*}{ Losing control } & $\bar{M}$ & 139 & 2.18 & 4.162 & \multirow{2}{*}{.608} & \multirow{2}{*}{.883} \\
\hline & $\mathrm{F}$ & 238 & 1.92 & 3.904 & & \\
\hline
\end{tabular}

*. $\mathrm{p}<.05, * * \mathrm{p}<.01$

\section{Acknowledgement}

This study was supported by research fund from Chosun University, 2015

\section{References}

[1] R. C. Kessler, P. Berglund, O. Demler, R. Jin, K. R. Merikangas and E. E. Walters, Lifetime prevalence and age-of-onset distributions of DSM-IV disorders in the National Comorbidity Survey Replication, Archives of General Psychiatry, (2005), Vol.62, No.6, pp.593-602.

[2] M. M. Weissman, Cross-national epidemiology of obsessive-compulsive disorder. CNS Spectrums, (1998), Vol.3, No.S1, pp.6-9.

[3] J. P. Hong, The survey of mental disorder in Korea 2016, Ministry of Health and Welfare, Seoul, (2017)

[4] Diagnostic and Statistical Manual: Mental Disorders(5th Ed), Ameican Psychiatric Association, Washington D.C. (2013).

[5] S. Rachman and P. de Silva, Abnormal and normal obsessions, Behaviour Research and Therapy, (1978), Vol.16, No.4, pp.233-248.

[6] D. A. Clark, J. Abramowitz, G. Alcolado, P. Alonso, A. Belloch, M. Bouvard, M. E. Coles, G. D. Hector, F. Alvarez, G. Garcia-Soriano, M. Ghisi, B. Gomez, M. Inozu, R. Moulding, A. S. Radomsky, G. Shams, C. Sica, G. Simos, W. Wong, Part 3: A question of perspective: The association between intrusive thoughts and obsessionality in 11 countries, Journal of Obsessive-Compulsive and Related Disorders, (2014), Vol.3, 
No.3, pp.292-299.

[7] L. M. Berry and B. Laskey, A review of obsessive intrusive thoughts in the general population, Journal of Obsessive-Compulsive and Related Disorders, (2012), Vol.1, No.2, pp.125-132.

[8] C. Purdon and D. A. Clark, Obsessive intrusive thoughts in nonclinical subjects, Part I: Content and relation with depressive, anxious and obsessional symptoms, Behaviour Research and Therapy, (1993), Vol.31, No.8, pp.713-720.

[9] H. J. Lee, Differences between autogenous obsessions and reactive obsessions in appraisal and control strategies, Masters Thesis, Seoul University, (1999) 